\section{The Relative Stability of the Alkali Metal Chelates of Dibenzoylmethane}

\author{
W. CONARD FERNELIUS AND LEGRAND \\ G. VAN UITERT \\ Department of Chemistry, The Pennsylvania \\ State University, State College, \\ Pennsylvania, U.S.A.
}

$\mathrm{B}^{\mathrm{r}}$ rändström ${ }^{1}$ has recently discussed the evidence bearing on the relative stability of the sodium and potassium chelates of $\beta$-ketoesters. He concludes that $\mathbf{K}+$ has a stronger tendency than $\mathrm{Na}+$ to give a chelate ring with the anion of a $\beta$-ketoester. Since none of the evidence previously available involves a direct measurement of these tendencies to form chelate rings, it seems worthwhile to consider some recent measurements in our laboratory on the tendencies of the alkali metal ions to form chelate rings with dibenzoylmethane (HCh) which is very closely related to the $\beta$-ketoesters.

By making potentiometric measurements in $75 \%$ dioxane-25\% water instead of pure water, it becomes possible to measure differences in chelate stability which are not measurable in water ${ }^{2-5}$. Thus, by using tetramethylammonium hydroxide as the titrating agent (the tetramethylammonium ion is a non-chelating ion), it became possible to make measurements which permit the calculation of formation constants (as defined by Bjerrum ${ }^{6}$ ) for the alkali metal chelates of dibenzoylmethane. The results of such measurements at $30^{\circ}$ $\left(4 \times 10^{-3} \mathrm{~mole}\right.$ of $\mathrm{HCh}$ and $1 \times 10^{-3} \mathrm{~mole}$ of $\mathrm{M}+$ in $100 \mathrm{ml}$ solution) expressed as $\log K$ are:

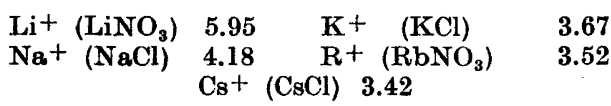

The order of variation with atomic num. ber from metal to metal is the same here as for the alkali earths and other periodic groups. Thus the direct measurements obtained here show that the tendency to form the sodium chelat $\theta$ is definitely greater than that for the potassium chelate.

1. Brändström, A. Acta Chem. Scand. 7 (1953) 223.

2. Van Uitert, L. G. and Haas, C. G., Jr. J. Am. Chem. Soc. 75 (1953) 451.
3. Van Uitert, L. G., Haas, C. G., Jr., Fernelius, W. C. and Douglas, B. E. J. Am. Chem. Soc. 75 (1953) 455.

4. Van Uitert, L. G., Fernelius, W. C. and Douglas, B. E. J. Am. Chem. Soc. 75 (1953) $457,2736,2739,3862$.

5. Van Uitert, L. G. and Fernelius, W. C. J. Am. Chem. Soc. 76 (1954) 375, 379.

6. Bjerrum, J. Metal Ammine Formation in Aqueous Solution, P. Haase and Son, Copenhagen 1941.

Received September 11, 1954.

A Remark to the Paper of W. C. Fernelius and L. G. Van Uitert on the Relative Stability of the Alkali Metal Chelates of Dibenzoylmethane ARNE BRANDSTROM

Research Laboratory of Pharmacia Ltd., Uppsala, Sweden

The very interesting measurements of Fernelius and Van Uitert ${ }^{1}$ on the rela. tive stability of the alkali metal chelates of dibenzoylmethane which were performed in a dioxane-water mixture are in good agreement with the evidence obtained by Brändström ${ }^{2-3}$ given in papers probably not yet accessible to Fernelius and Van Uitert. The paper of Brändström * cited by them deals with the behaviour in solvents of a much lower polarity (toluene and tert-butyl alcohol) where the situation may be quite different from that in a more polar medium ${ }^{3}$ and where unfortunately no exact measurements seem possible.

The interesting high value of the formation constant obtained with $\mathrm{Cs}+$ together with the fact that the alkali chelates appear to be of a pure ionic type might give rise to the question if the tetramethylammonium ion is an absolutely non-chelating ion.

1. Fernelius, W. C. and Van Uitert, L. G. Acta Chem. Scand. 8 (1954) 1726.

2. Brändström, A. Arkiv Kemi 7 (1954) 81.

3. Brändström, A. Arkiv Kemi 7 (1954) 181.

4. Brändström, A. Acta Chem. Scand. 7 (1953) 223. 